\title{
Algunas nuevas sobre Alphonso Hordognez ${ }^{1}$
}

\author{
Devid Paolini \\ The City College of New York
}

\section{RESUMEN}

En un artículo reciente, Gesiot (2019) ha mostrado cómo buena parte de la dedicatoria de la traducción italiana de Celestina es una adaptación, refundición y traducción de dos obras principales: el Decamerón y el Tirant lo Blanch. A las partes señaladas por el estudioso, indicamos algún pasaje más junto con unas consideraciones generales sobre lo que este descubrimiento significa con respecto al traductor de la obra maestra española.

Palabras Clave: Decamerón, Tirant, Hordognez, traducción italiana Celestina

\section{Some new information about Alphonso Hordognez}

\section{ABSTRACT}

In a recent article, Gesiot (2019) has showed that part of the dedicatory letter of the Italian translation of Celestina is an adaptation, recast and translation of two main works: the Decameron and Tirant lo Blanch. In addition to the passages already indicated by the mentioned scholar, we will point out other literary borrowings and some general considerations about what these new findings mean about the translator of the Spanish masterpiece.

Keywords: Decameron, Tirant, Hordognez, Italian translation of Celestina

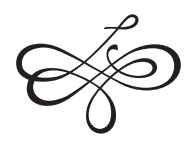

1.- Este trabajo se ha realizado en el marco del Proyecto de Investigación, Parnaseo (Servidor Web de Literatura Española), FFI2017-82588-P (AEI/FEDER, UE), concedido por el Ministerio de Economía, Industria y Competitividad. 
Unicuique suum, reza un viejo motto latino y como es justo que sea, hay que reconocer que las pocas ideas que siguen se han desarrollado a partir de un trabajo recién publicado (Gesiot 2019) cuyo contenido se repetirá aquí en parte (por lo menos en cuanto a las citas que recoge, añadiendo, por nuestra parte, unos pasajes más) por la importancia que tiene con respecto a Alphonso Hordognez. Como es bien sabido, Alphonso Hordognez es el enigmático traductor de Celestina al italiano, edición que vio la luz en Roma en enero de 1506 y que llevó a cabo a petición de Madonna Gentile de Campofregoso, hija ilegítima del famoso condotiero Federico de Montefeltro ${ }^{2}$. Su nombre aparece por primera vez en los materiales paratextuales que acompañan dicha obra. De hecho, en el título leemos ${ }^{3}$ :

Tragicocomedia de Calisto et Melibea, novamente agiontovi quello che fin qui manchava nel processo de loro innamoramento, nel quale se conthiene ultra il suo gratioso et dolce stilo assai pholosophiche sententie et advisi assai necessarii per gioveni, monstrando loro l'inganni che son renchiusi ne falsi servitori e rrofiane. Per Alphonso Hordognez, familiare de la sanctità di nostro signore Iulio Papa Secondo, ad instantia de la illustrissima madonna Gentile Feltria de Campo Fregoso, madonna sua observandissima, de lingua casteliana in italiana novamente per lo sopra dicto traducta. (31; la cursiva es nuestra)

Y, en la última octava con que se cierra la traducción, se nos dice:

Nel mille cinquecento cinque apunto

De spagnolo in idioman [sic] italiano

È stato questo opuscul transunto

Da me, Alphonso Hordognez, nato hispano,

A instantia di colei che in sé rasunto

Ogni bel modo et ornamento humano:

Gentil Felrria [sic] Fregosa, honesta e degna,

In cui vera virtù triumpha e regna.

(262; la cursiva es nuestra)

Todas las investigaciones que hasta ahora han intentado sacar a la luz alguna información adicional sobre Hordognez no han tenido éxito. Scoles (1961: 166), considerando que el traductor se nos presenta como «familiare» de papa Julio II, ha examinado los archivos vaticanos pero su

2.- Se nos permita reenviar a nuestro trabajo (Paolini 2011) para más información sobre Madonna Gentile.

3.- Todos los pasajes que se citarán vienen de la edición de Kish (1973). Los únicos cambios que se han aportado a esta son que se ha seguido el uso moderno en cuanto a la acentuación, el uso de mayúsculas y minúsculas, la distinción entre «u» $\mathrm{y}$ «V» y en cuanto a unión y separación de palabras. 
nombre no ha aparecido por ningún lado. La misma estudiosa ha adelantado, luego (169-75), algunas de las hipótesis que iba a presentar McPheeters (1961) en su investigación sobre Alonso de Proaza: el sucesor de este último en la cátedra de retórica en la Universidad de Valencia fue un tal Alfonso Ordóñez, involucrado, de diferente manera, en la impresión de diferentes tratados en latín a partir de 1518 , y podría tratarse del mismo personaje de quien estamos hablando aquí ${ }^{4}$. Lampugnani (2015: 5) en su estudio lingüístico sobre la traducción en cuestión, ha ampliado la búsqueda a los archivos Gonzaga y lo único que señala y que ha logrado encontrar es un grupo de epístolas escritas desde Faenza e Imola por un tal Alfonso Spagnolo, cuyo contenido no ha podido descifrar por la tinta que, prácticamente, se ha desvanecido con el tiempo. Por nuestra parte (Paolini 2011), en nuestro intento de esbozar un perfil biográfico cuanto más completo de la dedicataria de la traducción, Madonna Gentile de Campofregoso, tampoco hemos logrado hallar algún dato.

Estando así las cosas, de momento todo lo que podemos postular acerca de él viene de la obra misma y de las referencias, directas e indirectas, que allí se encuentran. Dejando de un lado lo que se puede conjeturar sobre su personalidad en base a la traducción llevada a cabo ${ }^{6}$, querríamos centrarnos aquí en dos pasajes del material paratextual con que se abre el texto: el poema y la carta dedicatoria.

En cuanto al primero ${ }^{7}$, ya Scoles (1961: 177 y sigs.) había llamado la atención sobre su forma, esta es, el soneto que indica claramente que Hordognez había llegado a Italia durante el pontificado de Alejandro VI, si no antes: ${ }^{8}$ como es bien sabido, esta forma métrica tardará todavía

4.- Estas son las conclusiones de Scoles - a quien McPheeters había adelantado sus descubrimientos por carta: «la grafia italianeggiante del cognome che troviamo nell'edizione di Nebrija, l'argomento 'e silentio' (nessuna notizia su Alfonso Ordóñez a Valencia nell'epoca in cui fu redatta la traduzione in Italia), insieme alla probabile formazione italiana dell'Ordóñez professore di retorica, non bastano, a mio giudizio, per trarre delle conclusioni definitive, ma rendono nell'insieme molto plausibile l'ipotesi che il traduttore e il professore di Valencia siano la stessa persona» (175).

5.- Aunque publicada en el año 2015, la investigación de Lampugnani es una puesta al día, aunque parcial, de su tesis de maestría terminada en 1983. Para más informaciones, véase nuestra reseña (Paolini 2018).

6.- Ya se han ocupado detenidamente de eso tanto Scoles (1961: 175-88) como Lampugnani (2015). A estos dos habría que añadir también la investigación de Gualano (2005-2006) que, lamentablemente, hasta el momento no nos ha sido posible consultar.

7.- «Ecco exequito donna il tuo precepto, / Ecco il comico tuo, tuo servitore, Et in sua compagnia, il dio d'amore, / Gratia, beltà, disio, speme, e suspecto. / Fede, perfidia, suon, canto, dilecto, / Suspir, vigilia, lacrime, dolore, / Caldo, freddo, pregion, forza, furore, / Iganni [sic], invidia, beffe, arte, e dispecto. / Lenoni, sdegno, buona e mala sotte, / E quel ch'al fin di lui sol si guadagna / Inimicitie, danno, infamia e morte, / Con altri effecti assai che non sparagna; / Ma se nel suo parlar ti parrà forte, / Scusal, che novamente vien di Spagna» (27).

8.- No hay que olvidar que Rodrigo de Borja, futuro papa Alejandro VI, se había marchado a Italia llamado por su tío, Alonso de Borja, cardenal desde 1444 y papa — con el nombre de 
unos años en desarrollarse en la península ibérica y delata, indirectamente, una estancia y presencia bastante larga de Hordognez en la península italiana - tanto como para familiarizarse con esta estructura que todavía no había arraigado en su país de origen?

Sin embargo, lo más interesante se encuentra en la carta dedicatoria que sigue al poema recién mencionado y que empieza así: "A la illustrissima madonna, madonna Gentile Feltria de Campo Fregoso, madonna sua observandissima» (28). Como ha mostrado Gesiot (2019), el paratexto mencionado es, en gran parte, una adaptación, traducción, refundición de dos obras conocidas en la época: el Decamerón de Boccaccio y el Tirant lo Blanch de Joanot Martorell (prínceps, Valencia, 1490; Barcelona, 1497). Y lo que no viene de las dos obras mencionadas - añadimos nosotros-es una paráfrasis del material paratextual ${ }^{10}$ de la traducción de Celestina que el mismo Hordognez recupera y reordena para incluirlo en su carta dedicatoria.

Veamos en la tabla a continuación este centón transcribiendo a la izquierda el texto completo de la dedicatoria y a la derecha las partes sacadas de la obra de Boccaccio, de Martorell y de los paratextos de la Celestina italiana. Para una comparación más efectiva, hemos decidido evidenciar en amarillo los pasajes iguales, parecidos o traducidos — que ya señala Gesiot (2019) —, en azul lo que hemos detectado nosotros - y que el investigador mencionado no registra-, y en verde los que vienen de la traducción de Hordognez:

Calisto III- desde 1455 hasta 1458, alrededor de la mitad del siglo xv para estudiar derecho canónico en la Universidad de Bolonia, así que Hordognez muy bien pudo haber llegado a Italia aun antes de 1492 (año en que Rodrigo fue elegido papa).

9.- Por supuesto, no tomamos en cuenta la calidad literaria en sí de la composición de Hordognez pero consideramos muy indicativo el hecho de que intentó escribir en una forma que a él resultaba — por lo menos al principio— casi seguramente totalmente extraña.

10.- Nos referimos, en particular, al subtítulo ("Tragicocomedia de Calisto et Melibea novamente agiontovi...», 31), la carta "Lo autore ad un suo amico» (33-4), el prólogo ("Dice Eraclito che tutte le cose in questo mondo... »,39-42) y el íncipit («Sequita la Tragicocomedia de Calista [sic] et Melibea...", 43). 
Illustrissima madonna: Come io son certo che V.S. multissime volte habia inteso che a veruna persona fa ingiuria chi honestamente usa sua ragione, natural cosa è adunque de ciaschuno che nasce sua vita quantunque può aiutare et conservare, e quella difendere con ogni astutia et sollicitudine guardandosi da li adversi casi che in questa nostra humana vita con assai nostro danno vedemo ogni giorno succedere. E questo si concede tanto, che alchuna volta è già advenuto che, per guardala [sic], senza culpa alchuna, si son commessi assai homicidii; et concedendo ciò le leggi (nele solicitudini dele quali è il ben vivere de ogni mortale) quanto maggiormente senza offesa d'alchuno a noi et qualunque altro è honesto ala conservatione nostra prendere quelli congrui remedii che noi possiano [sic].

Et quanto sia la presente opera spechio et chiaro exempio e virtuoso [sic] doctrina a nostro ben vivere, il nostro auctore per la presente opera chiaramente cel dimostra, insegnandoci li aguati et inganni di coloro che poco amore ci portano, quali per ogni minimo loro utile non curano a chi di loro si fida, con assai loro biasmo losenghevolemente ingannare, come nel processo di questi amanti compare.
Donne mie care, voi potete, così come io, molte volte avere udito che a niuna persona fa ingiuria chi onestamente usa la sua ragione. Natural ragione è, di ciascuno che ci nasce, la sua vita quanto può aiutare e conservare e difendere: e concedesi questo tanto, che alcuna volta è già addivenuto che, per guardar quella, senza colpa alcuna si sono uccisi degli uomini. E se questo concedono le leggi, nelle sollecitudini delle quali è il ben vivere d'ogni mortale, quanto maggiormente, senza offesa d'alcuno, è a noi e a qualunque altro onesto alla conservazione della nostra vita prendere quegli rimedii che noi possiamo? (Boccaccio, Decameron, introducción; véase Branca 1992, vol. 1: 32).

...les gestes e hystòries antigues dels hòmens forts e virtuosos, com sien spills molt clars, exemples e virtuosa doctrina de nostra vida, segons recita aquell gran orador Tuli. (Martorell, Tirant lo Blanch, Pròlech, f. a1v) ${ }^{11}$

... novamente agiontovi quello che fin a qui manchava nel processo de loro innamoramento

... monstrando loro l'inganni che son renchiusi ne falsi servitori e rofiane... (subtítulo Celestina italiana; Kish 1973: 31)

... Fatta similmente in adviso delli inganni delle ruffiane et mali et lusenghieri servitori. (íncipit $\mathrm{Ce}$ lestina italiana; 43)

11.- Citamos de la edición de la obra disponible en línea en la Biblioteca Virtual Joan Lluís Vives (Martorell 2006) 
Non per questo ali fraudulenti dala divina providentia fu, e ne sarà lor perdonato, mostrandoce quanta iustitia sua bontà comparte. E come $\mathrm{fu}$ in piacimento alo universal Creatore, che li cieli desseno influentia nel mondo e tenesseno dominio sopra la humana natura, donandoci diverse inclinationi di peccare et vitiosamente vivere, non per questo ne ha tolto il libero albitrio; che se quello è ben governato, vivendo virtuosamente, se può mitigare et vincere se usar volemo discretione.

Onde io mosso da tal consideratione, e vedendo la necessitate che tutti o la magior parte de questo presente tractato havemo, quale ci mostra apertamente via per la quale ci sapiamo guardare e difendere de l'iganni [sic] e losenghe de mali e tristi huomini; et anchora V.S., qualo [sic] mossa da virtuoso desiderio —non per miei meriti, ma per sua virtù-s'è degnata volerme pregare dovesse io tradure la presente tragicocomedia intitulata di Calisto et Melibea de lingua castigliana in italiano idioma, aciò che V.S. insieme con questa degna patria dove questa opera non è divulgata se possa alegrare di tante e così degne sentientie et avisi che sotto colore de piacevoleze vi sonno.
E per tant com la divina Providència ha ordenat e li plau que los VII planets donen influència en lo món e tenen domini sobre la humana natura, donant-los diversas inclinacions de peccar e viciosament viure, emperò no ls ha tolt lo universal Creador lo franch arbitre, que si aquell és ben regit les poden, virtuosament vivint, mitigar e vençre si usar volen de discreció (Martorell, Cap. 1, f. a2v)

...mi venne a la memoria non solamente la necessità che questa commune patria ha de la presente opera... de assai gratiose piacevolezze, ricordi et consigli contra lusinghieri e mali servitori et false donne factochiare... (carta Celestina italiana; 33 y 34)

...a vostra il-lustra senyoria sia stat grat voler-me pregar la giràs en lengua portoguesa... (Martorell, Dedicatòria, f. a1r)

...per ço que la nació d'on yo só natural se'n puxa alegrar e molt ajudar per los tants e tan insignes actes com hi són (Martorell, Dedicatòria, f. a1v)

... ultra il suo gratioso et dolce stilo assai pholosophiche sententie et advisi assai necessarii per gioveni... (subtítulo Celestina italiana; 31) 
Io, adunque, vedendo che legitima obligatione di ubidire suoi preghi mi costringe, quali a me sonno stati acceptabili commandamenti, e per satisfare in parte al desiderio che di servir quella continovamente mi sprona, meritamente me hanno obbligato a la executione di questa impresa, quantunque sia tenuto manifestare ogni opera virtuosa. Magiormente, che per il presente tractato, a quelli che lo legeranno, retenendo per sé le sententie necessarie et le lascivie lassando, grande utile ne venga.

E como già sia considerata mia insufficientia e le curiali e familiari occupationi, quali obstano ala adversità dela nobile fortuna, ${ }^{12}$ che non dan riposo a miei pensieri, che di questo travaglio iustamente iscusar mi possa.

Ma confidandomi nel superno Idio, donatore de tutti li beni, quale aiuta a li boni desiderii et suplisce ali diffecti di coloro che ben fa [sic] disiano, e porta boni propositi spesse volte nele mente, et in V.S. quale per sua virtù comportarà li errori così in stilo como in ordine, se per me fusseno posti inadvertentemente ne la presente traductione che veramente non nego, non vi se ne possa trovare, siando intrato in labirintho del quale me stesso a pena ne so trare. ...les quals pregàries són stades a mi molt acceptables manaments (Martorell, Dedicatòria, f. a1r)

...Molti van cappando le piacevolezze ... lassando leggermente passare quello che fa più al caso et utilità loro. Ma a quelli per li quali vero piacere è tutta ... reterranno la summa per loro utile, ridendo dele cose piacevoli, et le sententie et dicti de philosophi servaranno in lor memoria, per transporli in luochi convenienti a loro acti et prepositi. (prólogo Celestina italiana; 42)

E jatsia considerada ma insufficiència e les curials e familiars occupacions qui obsten, e les adversitats de la noÿble fortuna qui no donen repòs a la mia pensa, de aquest treball justament excusar-me pogués. (Martorell, Dedicatòria, fs. a1r-a1v)

Emperò, confiant en lo sobiran Bé, donador de tots los béns, qui ajuda als bons desigs supplint lo defalliment dels desijants e porta los bons propòsits a degudes fins, e vostra senyoria, qui per sa virtut comportarà los defalliments axí en stil com en orde, en lo present tractat per mi posats per inadvertència (Martorell, Dedicatòria, f. a1v)

12.- Hay aquí un interesante error que Hordognez traduce del Tirant y no corrige (pero sí se enmienda en algunas de las ediciones siguientes de la traducción de Celestina, como Milán 1514 y Milán 1515): la lección correcta era, seguramente, «movible» y no «noble». 
Per la qual cosa, suplico humilmente V.S. voglia acetarla como de servitore affectionato. Che se falimenti alcuni vi sonno, certamente madonna, parte ne a colpa la dicta lingua castiliana, quale in alchune partite, è impossibile posser ben tradure li vocaboli, secondo la affectione e desiderio che ho de servir V. Illustrissima S.

Non havendo io riguardo ala rudità de la ordinatione e differentia di sententie, a fine che per vostra virtù si communiche tra vostri parenti, amici e servitori, a ciò possano trarne il fructo che saper tiene, movendo lor cori a exequire ogni opera virtuosa, sprezando la iniquità de li vitii e la ferocità deli mostruosi acti, prendendo honorevoli partiti a conservatione di lor vite et honore.

Et aciò che di questa tragicocomedia lo primo auctore, né altri con epso non possa essere rimproperato, se errori alcuni li fusseno, como non dubito, V.S. voglia farli coreggere et amendarli, attribuendo la culpa di quelli a mio poco sapere et rudo ingegno et non al mancamento di mia voluntà desiderosa sempre di vostro servitio. Et aciò che li auctori per diffecto de miei falli non siano biasmati io solo voglio portarne il carico come per me solo sia stata traduta al commando di V.S., a la cui gratia humilmente mi riccomando. Vale.
Supplicant vostra virtuosísima senyoria accepteu com de servidor afectat la present obra -car si defalliments alguns hi són, certament, senyor, n'és en part causa la dita lengua anglesa, de la qual en algunes partides és impossible poder bé girar los vocables- attenent a la afectió e desig que contínuament tinch de servir vostra reduptable senyoria, no havent sguart a la ruditat de la ordinació e diferència de sentències, a fi que per vostra virtut la comuniqueu entre.ls servidors e altres perquè-n puguen traure lo fruyt que's pertany, movent los coratges de aquells e no duptar los aspres fets de les armes, e pendre honorosos partits endreçant-se a mantenir lo bé comú per qui milícia fonch trobada.

(Martorell, Dedicatòria, f. a1v)

E perquè en la present obra altri no puxa ésser increpat si defalliment algú trobat hi serà, yo, Johanot Martorell, cavaller, sols vull portar lo càrrech, e no altri ab mi, com per mi sols sia stada ventilada a servey de molt il-lustre príncep e senyor rey spectant don Ferrando de Portugal la present obra... (Martorell, Dedicatòria, f. a1v)

Ahora bien, ¿qué nos dice eso sobre Hordognez? Si miramos a la mención del Decamerón — texto muy conocido en la época — o a la paráfrasis que él mismo hace de algunos pasajes de su propia traducción, no mu- 
cho; pero si consideramos la otra obra en cuestión, sí algo. Primero, que al componer la carta dedicatoria de su traducción de Celestina tuvo a su alcance una copia del Tirant lo Blanch. Los pasajes que menciona y vierte al italiano son demasiado literales como para recordárselos de memoria y esto indica, indirectamente, que Hordognez como mínimo tuvo que haber pasado por unas de esas cortes donde la obra de Martorell era conocida y disponible —nos referimos, en particular, a Mantua, Ferrara y, tal vez, Urbino ${ }^{13}$, centro cultural, este último, en que residió durante mucho tiempo Madonna Gentile de Campofregoso (Paolini 2011). También hay que señalar, como ya ha recordado Gesiot (2019: 50), que el ejemplar de la prínceps del Tirant que se conserva actualmente en la Hispanic Society of America, proviene de los fondos de la Biblioteca Universitaria Alessandrina donde terminaron parte de los libros pertenecientes a Francesco Maria II della Rovere (1549-1631), último duque de Urbino. Segundo, al traducir del valenciano al italiano, Hordognez señala su posible procedencia, esta es, del levante ibérico peninsular ${ }^{14}$, hecho que sin duda fortalece la posibilidad de que haya llegado a Italia para buscar fortuna o, posiblemente, entrar al servicio del valenciano Rodrigo de Borja durante la segunda mitad del siglo $\mathrm{xV}-\mathrm{y}$, de todos modos, con anterioridad al pontificado de Julio I $^{15}$. También podría representar un punto

13.- Para la fortuna del Tirant en Italia y su traducción italiana, véanse Sansone (1980 y 1983), Annichiarico et alii (1984), Indini y Minervini (1990), Calvo Rigual (1992, 1994, 1997a, 1997b, 1997c, 2011a, 2011b y 2012), Martines (1993a, 1993b, 1997a, 1997b, 1997c, 2006, 2007 y 2012), Kolsky (1994), Barbieri (2011-12), Bognolo (2015), Concina (2015) y Gesiot (2015, 2017, 2018 y 2019). En Mantua, Isabella d'Este Gonzaga se interesó en la obra ya desde 1500 y tras encargar en 1501 una primera traducción al italiano a Niccolò da Correggio —cometido que no había todavía terminado a su muerte, en 1508-, le pidió luego a Lelio Manfredi que se pusiera con ello. Este último llevó a cabo la tarea entre 1514 y 1520 y la traducción se publicó, al final, póstuma, en 1538. En cuanto a las cortes señaladas (Mantua, Ferrara y Urbino), no hay que olvidar los lazos de parentesco que conectaban todas ellas: Isabella d'Este era la esposa de Francesco Gonzaga, señor de Mantua, y también hermana de Alfonso d'Este, señor de Ferrara; mientras que Elisabetta Gonzaga, hermana del señor de Mantua, era la esposa de Guidubaldo de Montefeltro, duque de Urbino.

14.- Como nos ha sugerido Di Camillo, si, como parece, Hordognez era originario del levante peninsular, entonces nos encontraríamos, en este caso — esto es, el de la traducción de Celestina-, frente a un contexto trilingüe en que un personaje cuyo idioma nativo era el valenciano tradujo una obra del castellano al italiano, es decir, tanto el idioma de partida como el de llegada eran a él ajenos. Aunque, por otro lado, tampoco puede del todo descartarse de que fuese un castellano con suficiente familiaridad con el valenciano o catalán.

15.- Además de la Roma papal, donde primero el pontificado de Calisto III (1455-1458) y luego el de Alejandro VI (1492-1503) trajeron a la ciudad eterna numerosos españoles, no puede pasarse por alto que hubo en la época también otro centro muy importante de atracción y consiguiente irradiación de la comunidad y cultura castellana, catalana y valenciana: nos referimos, en particular, al reino de Nápoles donde la dinastía de los Trastámara mantuvo el poder casi ininterrumpidamente desde 1442 (año en que Alfonso V el Magnánimo eligió la ciudad partenopea como capital de su reino) hasta bien entrado el siglo xvI. Podría también opinarse, ¿por qué no?, que Hordognez hubiese llegado inicialmente a Nápoles para pasar luego a Roma. Para un primer acercamiento a la cuestión - la presencia española en el sur 
a favor para la identificación del Alfonso Ordóñez, profesor de retórica en la Universidad de Valencia, con nuestro personaje - aunque personalmente tengamos muchas dudas al respecto ${ }^{16}$. Por último, querríamos llamar la atención sobre una remota posibilidad que se nos presenta: los pasajes que Hordognez tradujo e incorporó a su carta dedicatoria, ¿fueron simplemente algo que él encontró y consideró idóneos una tantum por lo que estaba haciendo, o más bien indican, por su parte, que tuvo la intención o el proyecto o el encargo de traducir él mismo el Tirant lo Blanch en italiano: ${ }^{17}$ Seguramente no es mucho lo que hemos podido añadir a lo ya conocido sobre Hordognez, sin embargo, como reza el dicho, algo es algo, y menos es nada.

y su irradiación en el resto de la península itálica, sobre todo en el norte- véanse, Cappelli (2004) y Gesiot (2016; 2018).

16.- Scoles, tras revisar los datos puestos a disposición por parte de McPheeters, había llegado a esta conclusión: «Professore di retorica ed editore: un intelletto particolarmente dotato, con una vasta preparazione oratoria, arricchita da una profonda conoscenza del greco e del latino; una figura eminente di umanista giunta all'acme della sua notorietà intorno al 1520: questo in sintesi è tutto quello che possiamo dire intorno ad Alfonso Ordóñez di Valencia» (1961: 174). Ahora bien, si se trata de la misma persona, no podemos sino reconocer el inmenso cambio que experimentó Hordognez en quince años (de 1505, año en que muy probablemente llevó a cabo su traducción, a 1520, año en que llegó a la "cumbre de toda buena fortuna» -retomando las palabras con que se cierra el Lazarillo de Tormes). Conscientes del hecho de que se necesitaría un análisis mucho más detenido y profundo de la cuestión, señalamos solamente un ejemplo que influye en nuestro escepticismo: en al acto 1, al hablar Celestina con Pármeno le dice esto: "Ma di, come maggiore, che a li audaci aiuta la fortuna» (72). El Hordognez «italiano» simplemente tradujo el error ("come maggiore») que se encontraba en su original castellano ("como mayor»), cuando la lectura exacta habría tenido que ser: «como [Publio Virgilio] Marón» ("Audentis Fortuna iuvat», Virgilio, Eneida, X, 284). Un profundo conocedor del latín, ¿no se habría dado cuenta del error y no lo habría corregido? Es verdad que Hordognez se apega mucho a la versión original, esto sí, pero pensamos — ¿tal vez ingenuamente? - que cualquier persona versada en latín habría sanado la corrupción.

17.- Cabría también la posibilidad de que lo que Hordognez incluye en su carta dedicatoria del Tirant formara parte de la traducción parcial que había llevado a cabo Niccolò da Correggio, tarea que había intentado el cortesano en cuestión como nos sugiere esta carta del prete da Correggio a Isabella d’Este Gonzaga: «Ill.ma patrona mia. Mio patrone [Niccolò da Correggio] e servitore de la S.V. avea dato principio a tradure Tirante, como vederà la S.V., ma non ge basta l'animo, perché dice che questo che vederà la S.V. è una colona e mezo e che 'I veneria alto due volte como l'è, ma el promete a la S.V. che l venirà e lo lezerà a la S.V. e che 'l non averà lete due carte che la S.V. lo intenderà como lui. Mando a la S.V. quello capitulo ve promesse; abenchè sia tempo d'altro che di componere pur ha comenzato quello capitulo promese a la S.V. ecc... Corrigiae, die 12 martii 1501» (Luzio y Renier 1893: 70-1; la cursiva es nuestra). 


\section{Bibliografía}

Annichiarico, Annamaria et alii, eds. Joanot Martorell, Tirante il Bianco, trad. de Lelio Manfredi. Roma: La Tipografia, 1984.

BARBIERI, Nicoletta Ilaria. Cultura letteraria intorno a Federico Gonzaga, primo duca di Mantova. Tesis doctoral. Università Cattolica del Sacro Cuore, Milán, 2011-2012. En línea.

Bognolo, Anna. "Il Tirante a Venezia. Sul contesto editoriale della prima edizione italiana (Pietro Nicolini da Sabbio alle spese di Federico Torresano d'Asola, 1538)». More about 'Tirant lo Blanc' / Més sobre el 'Tirant lo Blanc'. From the sources to the tradition / De les fonts a la tradició. Eds. Anna Maria Babbi y Vicent Josep Escartí. John Benjamins Publishing Company, 2015. 101-18.

Branca, Vittore, ed. Giovanni Boccaccio, Decameron. Turín: Einaudi, 1992.

Calvo Rigual, Cesáreo. "Il lessico della traduzione italiana del Tirant lo Blanc: studio contrastivo di due lingue romanze verso la fine del XV secolo e l'inizio del XVI». En Actas do XIX Congreso Internacional de Lingüística e Filoloxía Románicas. La Coruña: Fundación Pedro Barrié de la Maza, Conde de Fenosa, 1992. Vol. 2: 667-83.

-. "Iberismi ed italianismi nel Tirant lo Blanch e nella traduzione italiana di Lelio Manfredi». En Actas del VI Congreso Nacional de Italianistas. 1994. Vol. 1: 139-46.

—. "La selecció lèxica del català i de l'italià: el Tirant i la seua traducció italiana cara a cara». En Miscel-lània Germà Colón. Barcelona: Publicacions de l'Abadia de Montserrat, 1997a. 51-78.

-. "L'adaptació dels noms de lloc i de persona en el Tirant italià (1538)». En Butlletí Interior de la Societat d'Onomàstica (XXI Col-loqui de la Societat d'Onomàstica, IV Colloqui d'Onomàstica valenciana). Editorial Denes, 1997b. 233-54.

-. "Tirante il Bianco: aspectes de l'elaboració de la traducció italiana cinccentista del Tirant lo Blanc». En Actes del Col-loqui Internacional "Tirant Lo Blanch" l'albor de la novel-la moderna europea. Barcelona: Publicacions de l'Abadia de Montserrat, 1997c. 437-68.

-. "La versió italiana del Tirant». En La novel-la de Joanot Martorell i l'Europa del segle XV. Coord. Ricardo Bellveser. Institució Alfons el Magnànim, 2011a. Vol. 2: 693-720.

-. "Tirante il Bianco: la traducció renaixentista de Tirant lo Blanch». En "Tirant lo Blanch" políglota (1511-2011): cinc-cents anys de traduccions $i$ estudis. Coord. Vicente Martines. Marfil, 2011b. 41-3.

- Estudi contrastiu del lèxic de la traducció italiana del "Tirant lo Blanc» (1538). Institut d'Estudis Catalans, 2012. 
Concina, Chiara. "Ancora sulla fortuna del Tirant in Italia (con alcune postille sulla traduzione di Lelio Manfredi)». More about 'Tirant lo Blanc' I Més sobre el 'Tirant lo Blanc'. From the sources to the tradition / De les fonts a la tradició. Eds. Anna Maria Babbi y Vicent Josep Escartí. John Benjamins Publishing Company, 2015. 119-38.

Gesiot, Jacopo. "Il Tirant lo Blanch tra i versi del Mambriano di Francesco Cieco da Ferrara». Historias fingidas 3 (2015): 189-210.

-. "Indizi filologici dalla tradizione indiretta del Tirant lo Blanch». Medioevo romanzo 41 (2017): 390-413.

- Romanzi tiranni. La prosa iberica di cavalleria nel primo Cinquecento padano. Roma: Aracne Editrice, 2018.

-. "Ancora un plagio in Italia del Tirant lo Blanc: la lettera dedicatoria alla Tragicocomedia di Calisto e Melibea (1506)». Scripta 14 (2019): 48-56.

GUAlano, Andrea. Il plurilinguismo della prima traduzione italiana della "Celestina» (Roma, 1506). Tesis "di laurea", Univesità di Torino, 2005-2006.

KIsH, Kathleen V. An Edition of the First Italian Translation of the "Celestina". Chapel Hill: The University of North Carolina Press, 1973.

InDINI, Maria Luisa y Vincenzo MinerVINI. «Il viaggio di Tirante. Fortuna e infortuni di un romanzo cavalleresco». Romanica Vulgaria. Quaderni 12 (1990): 5-66.

Kolsky, Stephen. «Lelio Manfredi traduttore cortigiano: intorno al Carcere d'amore e al Tirante il Bianco». Civiltà mantovana s. 3, 29 (1994): 45-69.

LAmpUGnANI, Raffaele. Il metodo e lo stile di Alphonso Hordognez nella prima traduzione italiana della "Celestina". Tesis de maestría, Master of Arts from the School of Humanities, Flinders University of South Australia, 1983.

- La prima traduzione italiana de "La Celestina»: Primo commento linguistico e critico agli inizi del Cinquecento. Firenze: Olschki, 2015.

Luzio, Alessandro y Rodolfo Renier. «Niccolò da Correggio». Giornale Storico della Letteratura Italiana 22 (1893): 205-64.

Martines, Vicente. "El Tirant lo Blanc a Itàlia. «In Spagna è riputato come qui il Decamerone di Giovanne Boccacciom». Actes del novè Col.loqui Internacional de Llengua i Literatura Catalanes. Coords. Antoni Ferrando Francés, Lluís Meseguer y Rafael Alemany Ferrer. Abadia de Montserrat, 1993a. Vol. 2: 173-81.

—. "El Tirante il Bianco: èxit, ficció i ofici del Tirant lo Blanch a Itàlia». Actas do IV Congresso da Associaçâo Hispânica de Literatura Medieval. Coords. Aires Augusto Nascimento y Cristina Almeida Ribeiro. Cosmos, 1993b. Vol. 4: 331-4.

-. "Aspectes de la recepció de Tirant lo Blanch a partir de les seues traduccions espanyola, italiana i francesa dels segles XVI-XVIII». En Actas del VI Congreso Internacional de la Asociación Hispánica de Literatura Medieval. Coord. José Manuel Lucía Megías. Alcalá: Universidad, 1997a. Vol. 2: 997-1008. 
Martines, Vicente. "La versió italiana. El Tirante il Bianco (Venècia, 1538, 1566, 1611)». En El "Tirant» poliglota. Estudi sobre el "Tirant lo Blanch» a partir de les seues traduccions espanyola, italiana i francesa dels segles XVIXVIII. Coord. Vicente Martines. Barcelona: Publicacions de l'Abadia de Montserrat, 1997b. 53-84.

-. "El Tirant lo Blanch i les seues traducccions anteriors al segle XIX: Aspectes de recepció i de traducció». Caplletra: revista internacional de filología 23 (1997c): 91-118.

-. "Lectors i traduccions per a Tirant lo Blanch: Quixot, Renaixement, Barroc i Il-lustració per a la recepció de Tirant lo Blanch a Europa». En El "Quixot» i els clàssics valencians. Ed. Enric Balaguer. Alacant: Universitat d'Alacant, Vicerectorat d'Extensió Universitaria, 2006. 81-116.

-. "Recepció europea d'Ausiàs March i Tirant lo Blanch». En 2n Col·loqui Europeu d'Estudis Catalans. Coords. Alexander Fidora Riera y Eliseu Trenc Ballester. Montpellier: Association Française des Catalanistes; Péronnas: Editions de la Tour Gile, 2007. Vol. 1: 201-24.

-. "Les traductions de Tirant lo Blanch (1500-1800)». En Pour une histoire comparée des traductions: traductions des classiques, traductions du latin, traductions des langues romanes du moyen âge et de la première modernité. Coords. Dominique de Courcelles y Vicent Martines Peres. París: École des Chartes, 2012. 149-64.

Martorell, Joanot. Tirant lo Blanch. València, 1490. Transcripción en línea del ejemplar conocido como N1 o NY1. Alacant: Biblioteca Virtual Joan Lluís Vives, 2006

McPheteers, D. W. El humanista español Alonso de Proaza. Valencia: Castalia, 1961.

Paolini, Devid. "Madonna Gentile Feltria de Campofregoso, Alphonso Hordognez y la traducción italiana de La Celestina». eHumanista 19 (2011): 260-95.

- Reseña de: Raffaele Lampugnani, La prima traduzione italiana de "La Celestina»: Primo commento linguistico e critico agli inizi del Cinquecento. Firenze: Olschki, 2015. En Renaissance Quarterly 71.1 (2018): 387-8.

Sansone, Giuseppe E. "Lelio Manfredi traductor dels versos del Tirant lo Blanch». Estudis de Llengua i Literatura Catalanes 1 (Homenatge a Josep $M$. de Casacuberta, 1) (1980): 221-33.

-. "El Tirante de Lelio Manfredi». Estudis de llengua i literatura catalanes 6 (1983): 291-307.

SCOlEs, Emma. "Note sulla prima traduzione italiana della Celestina». Studi romanzi 33 (1961): 155-217. 
